\title{
APPLICATION OF BIOMETRICS IN AUTOMOTIVE INDUSTRY - CASE STUDY BASED ON IRIS RECOGNITION
}

\section{Edin Ćatović Saša Adamović}

${ }^{1}$ School of Computing and Informatics, Sinergija University,

Bijeljina, $\mathrm{BiH}$
Correspondence:

Edin Ćatović

e-mail:

cattovic@gmail.com

\begin{abstract}
:
Biometrics is used for unique identification of individuals. The identification can be done in two ways depending on the input, unique biological features or behavioral characteristics. Iris recognition is a method of biometric identification which is based on pattern recognition techniques. It provides high level of security and reliability, which makes it suitable for application in many areas. This method is intended to be used in one-to- many searching environment with stability as its main characteristics. The iris recognition method is based on high resolution images of the iris. It uses camera and IR illumination in order to eliminate or reduce reflection from the convex cornea and to obtain detailed rich images of very complex structure of the iris. Then, the images are converted into digital templates and stored in the biometric system database for future matching. In some cases, the efficiency of the iris recognition may be disrupted by eyeglasses or contact lenses, which can result in mismatching of the sample and the stored template. This paper deals with the implementation of the iris method in automotive industry in the area of safety and security.
\end{abstract}

Key words:

Biometrics, iris, security, recognition systems, automotive safety and security, eye tracking.

\section{BIOMETRICS}

Biometrics is a science that deals with measurable biological characteristics and/or behavioral characteristics. This term comes from the Greek words bios (life) and metron (measure). Standardized definition of biometrics is "automated identifying individuals based on their behavioral and biological characteristics" (Bidgoli, 2006). Some of the most common biometric physical characteristics include: fingerprints, hand geometry, iris, retina, facial features, thermograph face, vascular patterns (arrangement of veins), smell and DNA. Examples of behavioral biometrics include: signing, mouse tracking, walking and typing on the keyboard. Biometrics was regulated and defined in accordance with the ISO (International Organization for Standardization) requirements.

Fingerprint biometrics has a wide range of applications in the areas of civil society, military industry, legal proceedings, etc. One of the most important methods that biometrics heavily relies on is the Fingerprint 
Scanning. This biometric method is based on enrollment databases, which are used in protection of the international borders, criminal identification, and identity recognition. [1]

\begin{tabular}{|c|c|c|c|c|}
\hline Type & Stability & Accuracy & Signal acquisition & Applications \\
\hline $\begin{array}{l}\text { Physiological } \\
\text { characteristics }\end{array}$ & $\begin{array}{l}\text { Relatively } \\
\text { stable }\end{array}$ & Accurate & Easy & $\begin{array}{l}\text { Widely applicable to all } \\
\text { three functions of } \\
\text { biometrics }\end{array}$ \\
\hline $\begin{array}{l}\text { Behavior } \\
\text { characteristics }\end{array}$ & $\begin{array}{l}\text { Not very } \\
\text { stable }\end{array}$ & $\begin{array}{l}\text { Somewhat } \\
\text { accurate }\end{array}$ & Easy & $\begin{array}{l}\text { Mostly used for } \\
\text { authentication, or combined } \\
\text { with other biometrics for } \\
\text { authentication/identification }\end{array}$ \\
\hline $\begin{array}{l}\text { Psychological } \\
\text { characteristics }\end{array}$ & $\begin{array}{l}\text { Very } \\
\text { unstable }\end{array}$ & $\begin{array}{l}\text { Not } \\
\text { accurate }\end{array}$ & Very difficult & $\begin{array}{l}\text { It is still in the early stage of } \\
\text { development. Possibly used } \\
\text { for spoof protection in } \\
\text { extreme high security } \\
\text { environment }\end{array}$ \\
\hline
\end{tabular}

Fig. 1. Biometric characteristics comparison types

\section{IRIS AS AUTHENTICATION METHOD}

Iris recognition method is considered to be the most stable and reliable method of authentication. Therefore, for the protection of a car against stealing, or in order to prevent any manipulation with the car electronic system, the iris method can be implemented as a part of the system that is already integrated in the car. Iris system of recognition is an automated method that is used for identification. This method is based on mathematical pattern recognition techniques of high quality images. It scans one or both irises of an individual and the patterns are unique and very stable. [2]

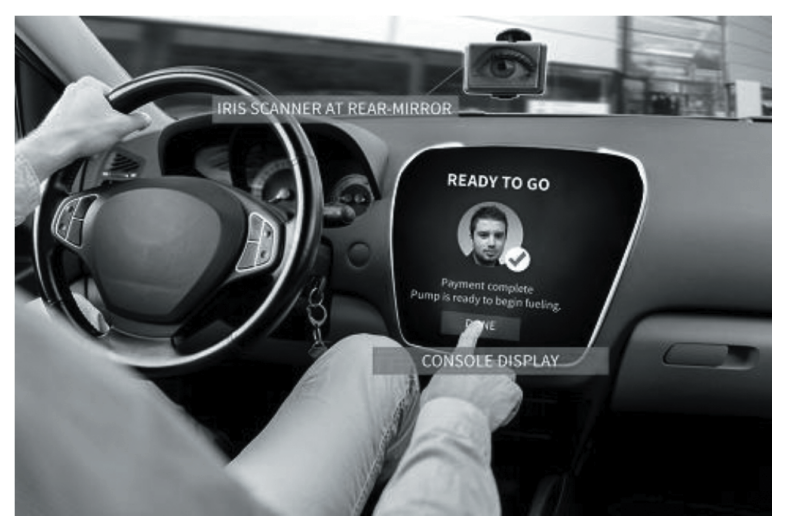

Fig. 2. Iris in a car, iris scanner in a rear view mirror; (source: https://venturebeat.com)

Characteristics of a well-implemented iris biometric system include extracting a great number of individual's unique features and a significantly reduced risk of storing the same templates for two or more individuals. In 2013, Apple integrated fingerprint sensors in their flagship phones and other companies soon started following the suit. In 2015, Fujitsu/NTT DOCOMO introduced iris recognition method in their smartphones which made it possible for the users to unlock their phones just by looking at them. Other big companies like Microsoft, Samsung, HP recognized the potential of this method and started integrating it in their products. Now, the question is why not integrate the same biometric method in connected cars?

Actually, we already have fingerprint recognition in connected cars. The users just need to touch a certain part of a car to complete the process of authentication. For example, it can be one of the doors or the ignition system. Where can the iris method be applied?

This method is intended to be implemented in connected cars in cases where we want to avoid physical contact. It is a kind of touchless biometric recognition. [3] Biometric technologies require a certain amount of cooperation from users that is, the users need to stand still while the devices scan their irises, faces, hands or fingers. Iris systems are typically used for distances shorter than $30 \mathrm{~cm}$ and in front of cameras. Therefore, during the process of recognition users must be motionless and look directly at the camera. As these devices are based on infrared illumination techniques, this type of recognition does not pose any health risks. [4]

\section{Biometric authentication of a driver}

Initially, the introduction of the iris method in automotive industry was to offer a new method of driver identification and authentication, as well as to create the opportunity to apply this method beyond the vehicle security and safety. Today, there is an increasing trend of ride-sharing services, which lead to the demand for the implementation of biometric technologies in terms of identification and authentication of a number of users who share the same car. A biometric method that enables a high level of security is the iris recognition method. It is usually installed in rear view mirrors and different car setups for different drivers can be adopted, for example, a limited speed setup in order to provide an increased level of safety for teenage drivers. Many big companies and organizations that frequently faced this issue have already implemented biometric technologies in order to be able to identify their drivers/employees at any time.

Besides the above-mentioned examples, payment services also use iris authentication. For example, Samsung 
Pay, Apple Pay and Google wallet are linked with credit cards and mobile phones which are used as payment terminals. Cars can have the same role. Once a car is connected to a system of payment, different kinds of biometric methods can be used to execute payments. For example, payments can be done at a gas station or in a coffee shop, or any drive-through. Also, there are in-car payments that are done via e-commerce.

In addition, this kind of biometric method can be used to increase safety. As its authentication process is very systematic and detailed, the iris recognition combined with CCD camera and algorithm based on Gabor Filter can prevent drunk drivers from starting their cars.

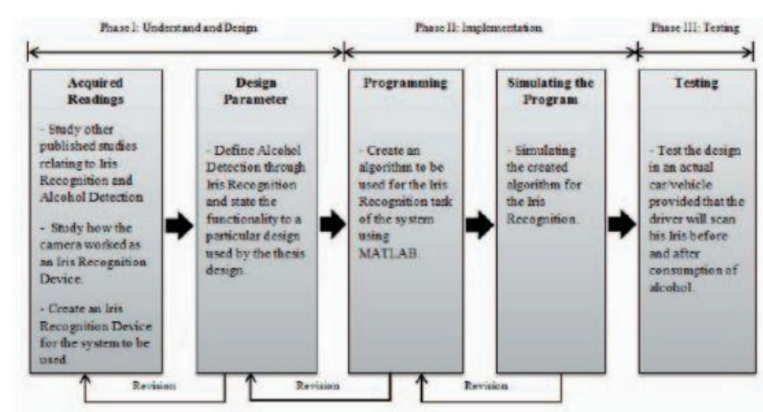

Fig. 3. Model of alcohol presence via iris recognition

In terms of safety in a car, iris recognition goes one step further by monitoring drivers' eyes for drowsiness and distraction. In newer cars, this system does not only alert drivers but also activates a predefined mode to drive the car to safety. Biometric technologies are also used for entertainment, such as setup of sound system, GPRS, cell phones, based on preferences of each driver.
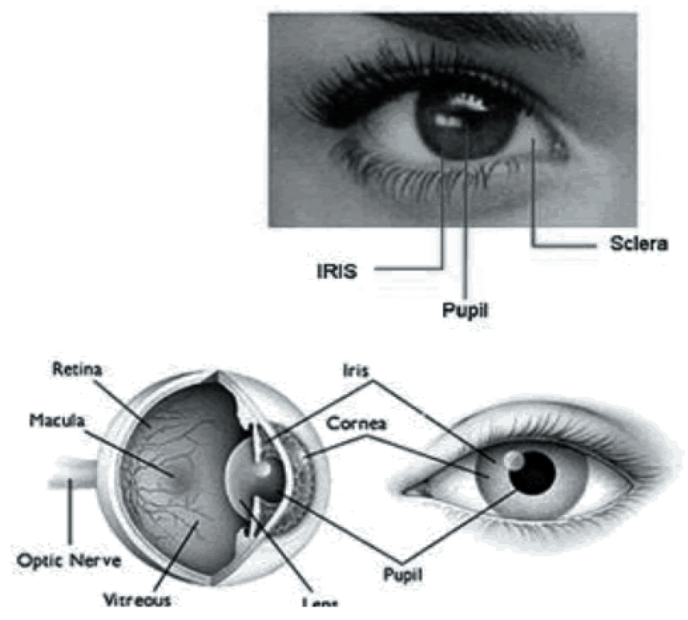

Fig. 3. Human eye structure

\section{IRIS RECOGNITION METHOD}

\section{Individuality}

There are no two irises alike, no correlation between iris patterns in twins and even the right and left eye of the same individual are different. An amount of information that can be measured in a single iris is much greater than the number of information taken from fingerprint. It is very difficult to modify texture of the iris by surgery. Also, it is rather easy to detect fake irises (designer contact lenses). While the general structure of the iris is genetically determined, particular aspects of its details depend on the circumstances. Human iris begins to form during the third month of pregnancy. By the eight month of pregnancy, the process of distinctive iris pattern creation is completed and the pigmentation lasts for several years after birth.

\section{Functionality}

Physical functionality of the iris is outstanding and it is often compared with the diaphragm (opening for passing light) of a camera. A normal iris has f-number - usually $f / 2$ or $f / 3$ - which is ideal for maximum exposure to light. If we compare it with a camera, this number would represent the diameter of a camera lens diaphragm opening. In other words, it represents the effective focal length of the lenses. For example, f/10 represents a diaphragm opening diameter that is one tenth of the focal length or the focal length is the length of ten openings.

The time that iris needs for changing the amount of light it receives is about fifth of a second. On the other hand, the reduction of the light is minor, less than factor of 20 (one more f-stop). This leads to a conclusion that the iris is not responsible for the control of light intensity, which is the function performed by the rods and cones in the back of retina.

Just like changing f-stop on a camera, the iris can effectively reduce aberrations, especially in bright conditions and increase the depth of field.

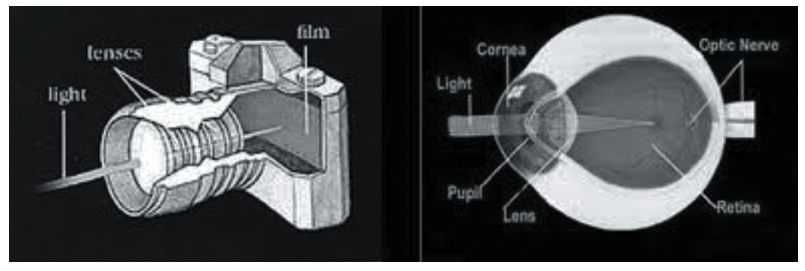

Fig. 4. Comparison: camera vs human eye (iris) 
In conclusion, the individual components of the eye function in a manner similar to a camera shown in a [Figure 4.]. Cornea function is similar to a camera lens cover. As the main focusing element of the eye, cornea receives a wide range of light rays, and bends them through the pupil. The pupil is a dark round opening in the center of the colored iris. The iris and pupil function like an opening of the camera. The function of the eye lens is to focus light on the back of the eye, just like the lens in a camera. The retina is placed on the back of the eye and its role is the same as the role of a film in a camera i.e. image sensor in digital cameras. The retina can be described as a membrane which contains photoreceptor nerve cells that are placed inside the back wall of the eye. These photoreceptor nerve cells of the retina transform light rays into electrical impulses. The optic nerve transmits the electrical impulses to the brain. The Macula is responsible for sharp vision, for example, when we read or watch something that requires focus, while the peripheral retina is responsible for peripheral vision.

\section{Characteristics}

Iris recognition technology converts visible iris characteristics into a 512 byte IrisCode, a template which is stored into biometric system database for future verification. Speaking of storage terms, 512 bytes is a small size for a biometric template but it stores a large quantity of information, which makes it quite unique. This amount of information is massive. For example, from iris with diameter of $11 \mathrm{~mm}$, Daugman's algorithms provide 3.4 bits of data per square $\mathrm{mm}$. Based on the density of information like this one, it can be concluded that each iris has 266 unique points. In comparison, the traditional biometric fingerprint technology stores 13-60 points. Daugman concludes that 173 independent binary degrees-of-freedom can be extracted from his algorithm, which is a significant number for biometrics.

\section{Algorithms}

The first step for biometric system based on iris is to locate the eye with the camera. The distance between the eye and a camera should not be greater than $90 \mathrm{~cm}$ (ideal distance is between $30-40 \mathrm{~cm}$ ). After the eye is captured by the camera, the algorithm scans the iris from right to the left and locates its outer edge. The horizontal approach is adopted because of a possible obstruction by the eyelids. At the same time, the camera locates the inner edge of the pupil, excluding points that are 90 degrees or more in relation to the shortest line between the eye and the camera, which is caused by lighting issues and inherent moisture. [5]

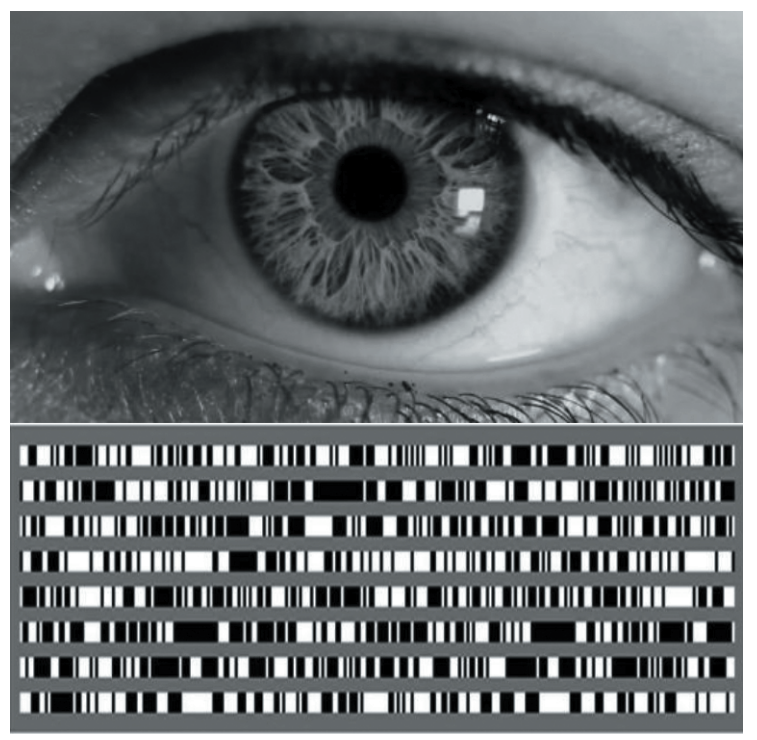

Fig. 5. Algorithm of Iris Area

Monochrome camera uses both types of light, visible and infrared. The infrared light is located within 700-900 $\mathrm{nm}$ range. The algorithm uses 2-D Gabor wavelets for mapping and filtering segments of the iris, in the form of vectors. (phasors). This operation does not include the entire surface of the iris, but rather its portion at the top. Also, 45 degrees of the iris bottom is not included because of the eyelids and camera light reflections. In future authentications and identifications, the biometric system will not use images to compare irises, but hexadecimal representations of the data returned by wavelet mapping and filtering. [9]

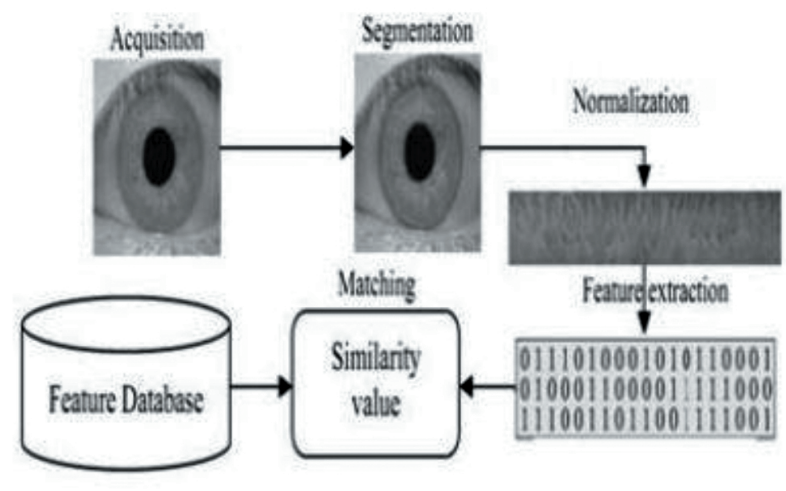

Fig. 6. Block diagram of iris recognition (algorithm) 


\section{IRIS RECOGNITION MATLAB SIMULATION}

MATLAB is a high level interactive environment for numerical computing, visualization and programming. Via MATLAB we can analyze data, create models and applications, as well as develop algorithms.

The iris recognition can be successfully simulated by using this software. The Image Processing Toolbox, which is available in this software, enables us to detect, normalize and match templates with the database.

The main functions of Image Processing Toolbox are:

- Filtering, deblurring, and enhancement of the image;

- Analysis of the image, including morphology, segmentation, measurement and feature extraction;

- Intensity-based image registration and spatial transformation methods;

- Image transformations;

- Interactive tools, which include Region Of Interest (ROI) selections, histograms, and distance measurements.

Array is a basic data structure in MATLAB. The representation of an image is in a form of real-valued ordered sets of color or intensity data. Most images in MATLAB are stored as two dimensional arrays, in which each element of the matrix corresponds to a single pixel in the image which is displayed. [6]

An image is taken by a standard camera which is usually placed in a rear view mirror. Once the camera captures the face of an individual, the iris recognition algorithm does the segmentation of face image to acquire only the iris region, separate from the pupil region. At the moment when iris edges are detected, using Canny Edge Detection method, iris image is transformed to the digital form, and matched with values stored in database.

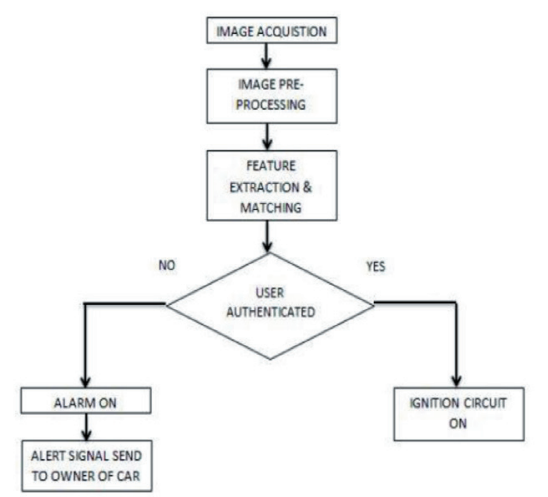

Fig. 7. Flowchart diagram, iris recognition connected with car ignition
Canny Edge Detection Method is based on finding the edges of iris, by looking for local maxima of the gradient of I. The "I" represents intensity of the image, while the gradient is calculated by using the derivative of the Gaussian filter.

By using two thresholds, this method detects strong and weak edges and includes the weak edges in the output only if they are connected to strong edges. [7]

Canny Method is:

$\mathrm{BW}=$ edge (I, 'canny')

BW = edge (I, 'canny', THRESH)

Where THRESH is vector which includes two elements, first low threshold and second high threshold.

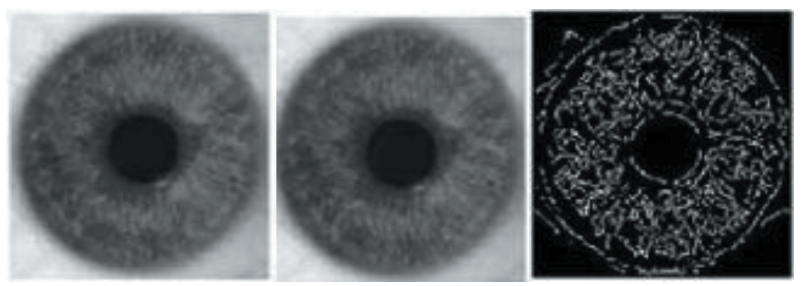

Fig. 8. Original, grayscale and canny edge image

The iris image is taken first and it is converted to a grayscale image for image pre-processing. Then, the Canny Edge detection function is applied and the template with edges of iris and pupil is formed. (8)

\section{CONCLUSION}

One of the goals of this analysis is to present the areas in which the iris recognition method can be used in the coming years.

In order to make biometrics more applicable and acceptable researchers are developing new techniques. For example, iris recognition systems that can be used even from a greater distance and while users are in motion.

This paper presents new multidisciplinary technologies for iris recognition that are less constrained than the traditional biometric systems. In order to understand and implement these technologies a multidisciplinary approach is required. The described technologies include all aspects of iris recognition systems including methods of acquiring and processing biometric data.

The following characteristics make iris recognition method attractive:

- Very stable: pattern of human iris remains unchanged throughout lifetime 
- Flexibility: easy to integrate in the existing security systems or it can operate as a standalone system

- Unique: probability of two identical irises is insignificant

- Non-invasive: iris recognition is contactless and it offers a high level of accuracy

- Reliable: a distinctive iris pattern is not susceptible to theft, loss or compromise.

- Versatile: it can work in one-to-many and oneto-one matching or verification which makes it ideal for multifactor authentication systems

- Performance: suitable for environments with large databases without slowing down the system

Model of described Iris Recognition System can be integrated into the Electronic Control Unit (ECU) of the automobile to ensure the security of a vehicle in case of theft. Further analysis might be focused on a detection of the level of drowsiness in drivers and the same algorithm is possible to be used. This system would identify the level of closeness of drivers' one or both eyes and alert the driver to take an appropriate action. Such a biometric system could increase the safety of drivers as well as improve the overall safety of other traffic participants.

\section{REFERENCES}

[1] Sciencedirect.com, "A New Texture Analysis Approach for Iris Recognition," 2015.

[2] Y. D. a. C. B. Z. Zhou, Transforming traditional iris recognition systems to work on non-ideal situations, 2009.

[3] V. G. a. V. A. R. Garg, A Drowsy Driver Detection and security system, 2009.

[4] J. S. D. a. L. J. Fridrich, Detection of Copy- Move Forgery in Digital Images, 2003.

[5] D. Zang, Automated biometrics technologies and systems, Boston, Mass, USA, 2000.

[6] H. Bidgoli, Handbook of Information Security, Threats, Vulnerabilities, Prevention and Management, 2006.

[7] W. WW, Overview of research on driver drowsiness definition and driver drowsiness detection, Munich, 1994.

[8] J. Russ, Forensic Uses of Digital Imaging, O. J. Simpson's skin was darkened in a police photograph, 2001.

[9] Johnson, V.; Farid, H;, Exposing Digital Forgeries by Detecting Inconsistencies in Lighting, New York, 2005. 